\title{
Photometric periods of LQ Hydrae between 1992 and 2000
}

\author{
J. You
}

\author{
Astronomy Division, Department of Physical Sciences, PO Box 3000, 90014 University of Oulu, Finland \\ e-mail: jianpeng.you@oulu.fi
}

Received 9 May 2007 / Accepted 23 August 2007

\section{ABSTRACT}

\begin{abstract}
Aims. Stellar spots produce periodic variations in photometric data. We aim to determine the observed periods with a high accuracy to improve measurements of stellar differential rotation.

Methods. We applied the method of three-stage weighted multichannel period analysis (MPA) to photometric observations of LQ Hydrae obtained in 1992-2000.

Results. We detected two periods in 10/1993-5/1994, two periods in 10/1998-6/1999, and three periods in 10/1999-3/2000. The primary period was found to be present for all observing seasons with small fluctuations within 1.60-1.61, while including the additional secondary periods, the fluctuations become three times larger within $1.585-1.625$. We also found that the amplitudes of the model curves of the primary period varied and reached their maxima in 10/1993-5/1994 and their minima in 10/1997-6/1998, while the amplitudes of the additional periods were always below the amplitudes of the primary period in the same season.

Conclusions. We conclude that the stellar spots responsible for the photometric variations of the primary period are long-lived and change their positions between two latitudes corresponding to two different rotation periods of $1^{\mathrm{d}} .60$ and of $1^{\mathrm{d}} 61$ (or have slightly varying longitude migration rates). The constancy of the primary period in the individual seasons means a constancy of latitude over the observation time intervals. We interpret the appearance of the additional periods to be caused by the formation of new surface structures at different latitudes. The surface structure is thus temporary. Based on our detected period range, the corresponding latitudinal differential rotation rate $\Delta \Omega / \Omega=0.025$ is small compared with that of the $\operatorname{Sun}(\Delta \Omega / \Omega=0.2)$.
\end{abstract}

Key words. method: data analysis - stars: rotation - starspots - stars individual: LQ Hydrae - stars: activity - starspots

\section{Introduction}

Differential rotation and convection, drive stellar magnetic activity and control the intrinsic dynamo processes. Kitchatinov \& Rüdiger (1999) developed a differential rotation model for global circulation in the outer stellar convection zone and derived the related surface differential rotation for two spectral classes G2 and K5, based on the dynamo theory of stellar activity. Measurements of stellar differential rotation depend on tracking large magnetic activity spots on the stellar surface. Using photometric modeling of stellar surface spots it has been possible to derive differential parameters, such as the differential coefficient and the equatorial rotation period, and thus to constrain the absolute range in latitude of the surface spot region (see the recent study of $\kappa^{1}$ Ceti by Walker et al. 2007 and of $\varepsilon$ Eri by Croll et al. 2006).

Spectral deconvolution such as Doppler imaging to resolve stellar surface temporal evolution is one of the important techniques to study stellar differential rotation. In this framework, Petit et al. (2004) described the main modeling tools and summarized the results for a small number of cool, active, fast rotating stars. Also Jeffers et al. (2007) analyzed a long term temporal evolution of differential rotation for an active star $\mathrm{AB}$ Doradus. With these techniques it is also possible to determine the dependence of starspot rotation rate as a function of latitude (Barnes et al. 2005; Marsden et al. 2005).

LQ Hydrae (HD 82558; average $V=7$. $8, B-V=0$ m.9; $\mathrm{K} 2 \mathrm{~V}$ ) is a single dwarf, and is one of the rapidly rotating stars for which the differential rotation has been determined. It is believed to have just arrived at the zero-age main-sequence (Fekel et al. 1986). The star has been studied extensively in the past. Fekel et al. (1986) and Eggen (1984) were the first to draw attention to it.

For a period analysis of LQ Hydrae, Fekel et al. (1986) determined the photometric light variations of LQ Hydrae and found an amplitude of 0.1 in $V$ and a period of $P_{\text {rot }}=1$ d.66. From the rotation period and a $v \sin i=25 \mathrm{~km} \mathrm{~s}^{-1}$ they derived an inclination of $70^{\circ}-90^{\circ}$. Jetsu (1993) used all the photometric data available at that time to derive a long-term photometric period of $P_{\text {rot }}=1.601136 \pm 0.000013$ based on the normalized $B$ - and $V$-light curves. Strassmeier et al. (1997) performed a period analysis on all available data since the discovery of the light variations of LQ Hydrae and found a long-term average photometric period of $P_{\text {rot }}=1.60088 \pm 0.00003$ from the combination of 12 years of $V$-band data. Berdyugina et al. (2002) analyzed photometric observations spanning almost 20 years and refined the rotation period to be $P_{\text {rot }}=1.601052 \pm 0.000014$. They also found a slight differential rotation of $\Delta \Omega / \Omega=0.002$ with $P_{\min }=1^{\mathrm{d}} 600905$ and $P_{\max }=1.601198$. Messina \& Guinan (2003) suggested cyclic changes in the period of LQ Hydrae, in a range of $P_{\text {rot }}=1.5938-1.6154$. The long term cycle length in their analysis was 6.2 years based on their time series analysis of photometric data spanning about 18 years. Kövári et al. (2004) refined the rotation period to $P_{\text {rot }}=1.60066 \pm 0.00013$ from photoelectric observations over 21 years. Kövári \& Weber (2004) used two imaging methods to analyze the differential rotation of LQ Hydrae. They found that the star has an almost rigid rotation with weak equatorial acceleration of the differential rotation of $\Delta \Omega / \Omega=0.0056$. McIvor et al. (2004) used a rotational period of 1.61 to do Zeeman-Doppler mapping of the surface magnetic field and found that the global magnetic field structure can change dramatically in one year. 
The period analysis of LQ Hydrae is the basis for studying different aspects of this single Sun-like star. All rotational periods so far derived are different, though close to 1.6. We suspect that the apparent rotational period, dominated by spots at different latitudes, varies with time. Therefore, any derived, constant, long-term period is likely to be affected by the star's seasonal short term physical activities. In this paper, we re-analyze the periods of LQ Hydrae and explore its surface differential rotation.

\section{Observations}

The UBV photometric data of LQ Hydrae used in this study were obtained at the Mount Hopkins Observatory. The photometry was carried out between October 1992, and March 2000, with the 10-inch Automatic Photoelectric Telescope (APT), from which data until 1993 were also used by Jetsu (1993). A description of the APT observing and reduction routines can be found in Boyd et al. (1984). External errors of 0.013 and 0.012 in $B$ and $V$ filters respectively have been reported by Boyd et al. (1990). Because the observations with an internal error greater than 0 . 02 are automatically rejected by the APT, the U channel has the highest rejection rate. Jetsu (1993) used only the B and V because of the high rejection rate in $U$. In the new data used in this paper, the $U$ channel has almost as many points as the B and $\mathrm{V}$ channels. Thus, we include all three channels in our time series analysis. In Table 1, we give the time span and the number of observations in the $\mathrm{U}, \mathrm{B}$, and $\mathrm{V}$ channels for each season.

The external errors of 0.013 and 0.012 are first used for weighting the $\mathrm{B}$ and $\mathrm{V}$ in our preliminary analysis of the data for determining the correct errors in U. Based on the values of the reduced $\chi^{2}$ of the three individual channels, the error in $U$ was chosen as 0.015 . Thus, the errors of $0.015,0.013$ and 0.012 are used for weighting the data in the U, B and V channels respectively, whereas all data in the same channel have the same weight.

\section{Methods and results}

We used the method of multichannel weighted period analysis (MPA, You et al. 2000) to search the rotation periods of LQ Hydrae. The method finds common periods in all channels simultaneously. The three consecutive stages in the full algorithm are phase dispersion minimization (PDM), linear modeling (LM) and nonlinear modeling (NLM). The first application of MPA, to HN Peg, has been presented in You \& Duemmler (2005).

Stellar activity indicators (spots or spot groups) evolve with time, and new spots may appear at different latitudes, possibly with altered rotation period if significant differential rotation exists. Therefore, there may exist multiple periods, period and amplitude changes, and phase shifts in the light curves. To allow for these effects we analyzed the data separately for each season.

In the first stage of our analysis we applied PDM simultaneously in $\mathrm{U}, \mathrm{B}$ and $\mathrm{V}$ : we confirm the previous finding that the primary rotation period for this star is around 1.6. A significant period is refined in LM and is finally optimized in NLM (see below, You et al. 2000; You \& Duemmler 2005). The LM and the NLM use the same sinusoidal model of the second order for modeling the data. The sinusoid functions in the fitting model are chosen up to the second order, because of the probable complexity of the physical light curves. The model for fitting the data with only one period is

$$
\begin{aligned}
M^{c}\left(t, \beta^{c}(P)\right)= & A_{0}^{c}+A_{1}^{c} \cos (2 \pi r f t)+B_{1}^{c} \sin (2 \pi r f t) \\
& +A_{2}^{c} \cos (4 \pi r f t)+B_{2}^{c} \sin (4 \pi r f t),
\end{aligned}
$$

where $f=1 / P$ is the trial frequency and $\beta^{c}(P)$ includes all linear parameters $A_{0}^{c}, A_{1}^{c}, B_{1}^{c}, A_{2}^{c}, B_{2}^{c}$. Here, $A_{0}^{c}$ is the mean magnitude and $A_{1}^{c}, B_{1}^{c}, A_{2}^{c}, B_{2}^{c}$ are the amplitudes of the cosine and sine functions for channel $c(\mathrm{U}, \mathrm{B}$ and $\mathrm{V})$. For the full multichannel model, all $\beta^{c}$ of the individual channels are independent, but the period $P$ is the same in all models $M^{c}$ fitted to the full set of observations. Here, we use only the periods from the combined multichannel analysis, based on the requirement that the real periods are not wavelength dependent and the fact that the multichannel periods are more accurate than any of the single channel periods (You et al. 2000).

The identification and the final optimization of a second significant period is performed by analyzing the residuals, which are obtained in the NLM stage by subtracting the first significant period. If a second significant period is found in the residuals, it is refined again using NLM to the residuals. This is slightly different from the procedure of the final optimization of additional periods in You \& Duemmler (2005) where only the original data are used. This is because for LQ Hydrae the periods are so close to each other that they can not be always separated into different minima in the LM spectra. While the second period is refined from NLM using the first residuals, the second residuals are also obtained by subtracting the second significant period from the first residuals. Then the second residuals are analyzed again in order to study a possible third period. The procedure of period identification is repeated until no significant minima are left in the LM spectrum.

Figure 1 shows the multichannel WRSS $(f)$ (weighted residual square sum) spectra for season S2. For convenience, the spectra are plotted, not as a function of the trial frequency $f$, but as a function of the trial period $P$. The top spectrum is derived from the original data and reveals the period of 1.60263 as the deepest minimum, while two minima at 1.23 and at 1.45 are identified as spurious, arising from the combination of the primary period with the periodicities in the data sampling and the higher harmonics of the model (You et al. 2000). Here, the primary period is the first detected period which has the largest amplitude (see later) if more than one period is present in a given season. The central spectrum is derived from the residuals of S2 after the NLM fit, where a deep minimum at 1.62465 is seen. The bottom spectrum is obtained by subtracting the second period from the central spectrum. Here, it contains only noise and thus our MPA is complete. Our detection of the two periods is further supported by the phase process diagrams (PPDs) of the two periods in Fig. 2. The three PPDs on the left are created by folding the original data in S2 with the primary period of 1.60263 . The three PPDs on the right are created with the second period of 1.62465 by folding the residuals after subtracting the first period from the original data. All phases in the PPDs have been computed with $\phi=0$ at HJD $=2440000$. Although not as clear as the PPDs on the left, the PPDs on the right still show a pattern, with a recognisable maximum and minimum.

Figure 3 shows the multichannel LM spectra for S6. The top spectrum reveals the primary period of 1.60830 . The central spectrum is obtained from the residuals after the NLM fit. Here, the second significant period of 1.58658 is visible, while two minima on the far left of 1.00 and on the far right of 1.99 are identified to be spurious arising from the data grouping and the 

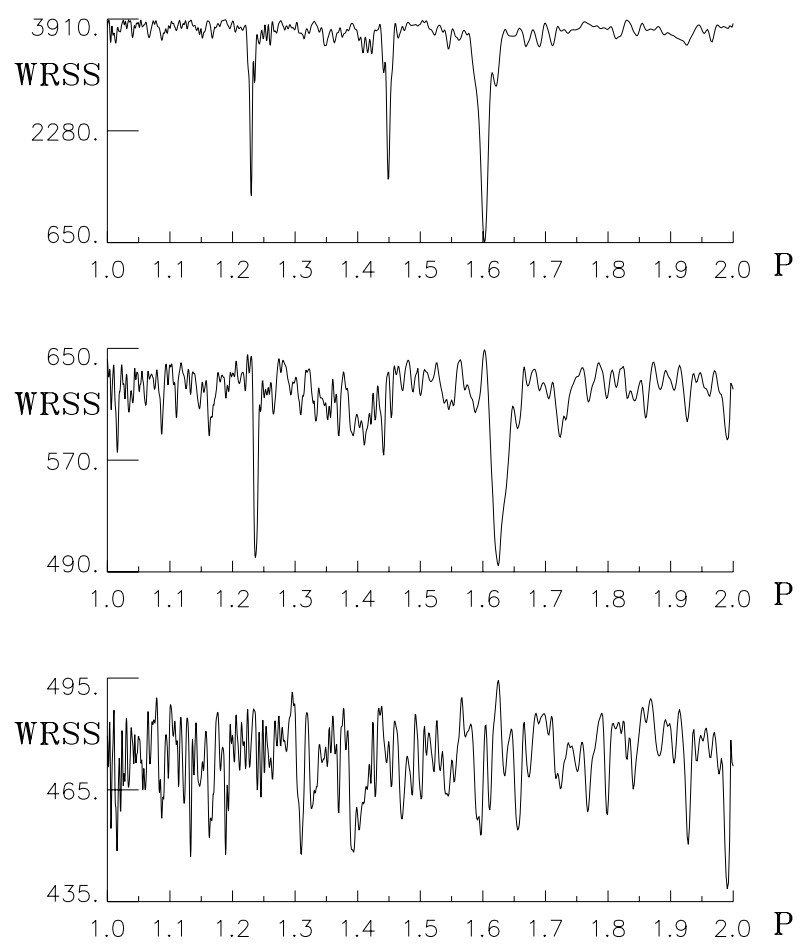

Fig. 1. The multichannel (UBV) weighted residual square sum (WRSS) LM spectra of season S2. The spectra are plotted with the trial periods $P$ in days. The top spectrum is derived from the original data showing the period of 1.60263 (the two minima at 1.23 and at 1.45 are spurious). The central spectrum is derived from the residuals after subtracting the first period, showing the second minimum of 1.62465 . The bottom spectrum is obtained by subtracting the second period from the central spectrum and shows only noise.
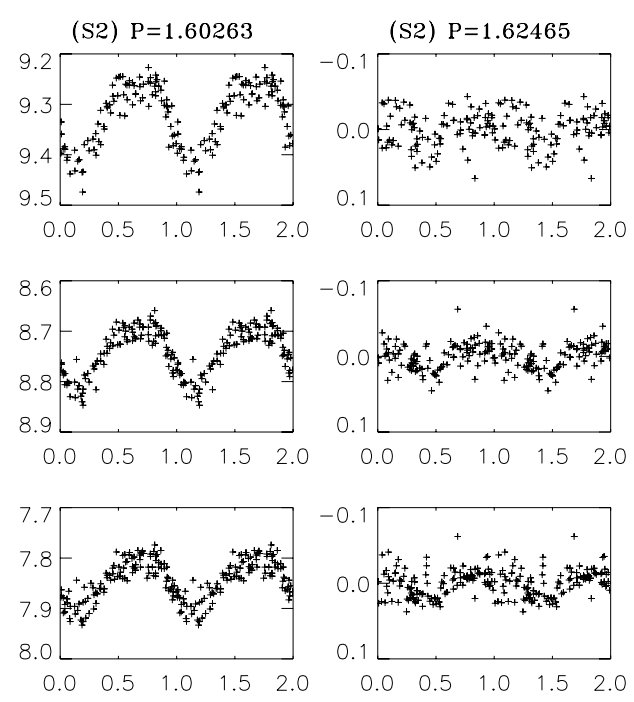

$(\mathrm{V})$

Fig. 2. Phase process diagrams (PPDs) of season S2. The left three PPDs are created by folding the original data with the primary period of 1.60263 . The right three PPDs are created with the second period of 1.62465 by folding the residuals after subtracting the first period from the original data. All phases in the PPDs have been computed with $\phi=0$ at $\mathrm{HJD}=2440000$.

noise fluctuations. The bottom spectrum is obtained by subtracting the second period from the central residual spectrum. Here, except for the two spurious deep minima on the far left and on the far right, the whole spectrum shows only noise. Thus, there is no third period. The two detected periods are checked with
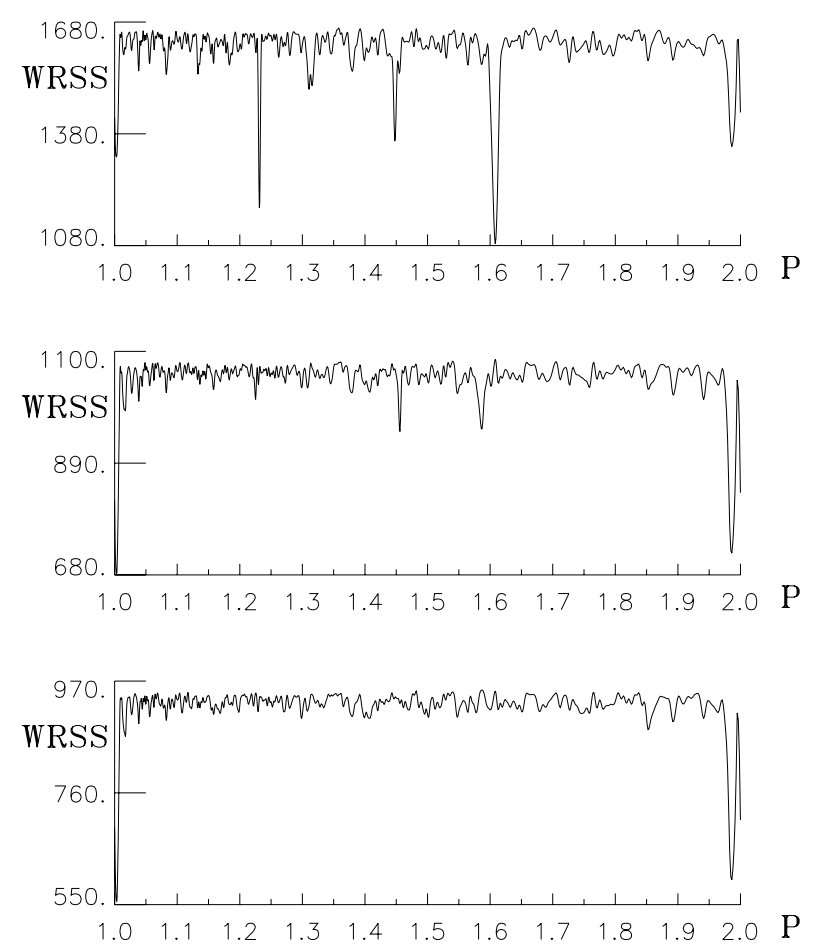

Fig. 3. The multichannel (UBV) LM spectra of season S6. See text and Fig. 1 for explanation.
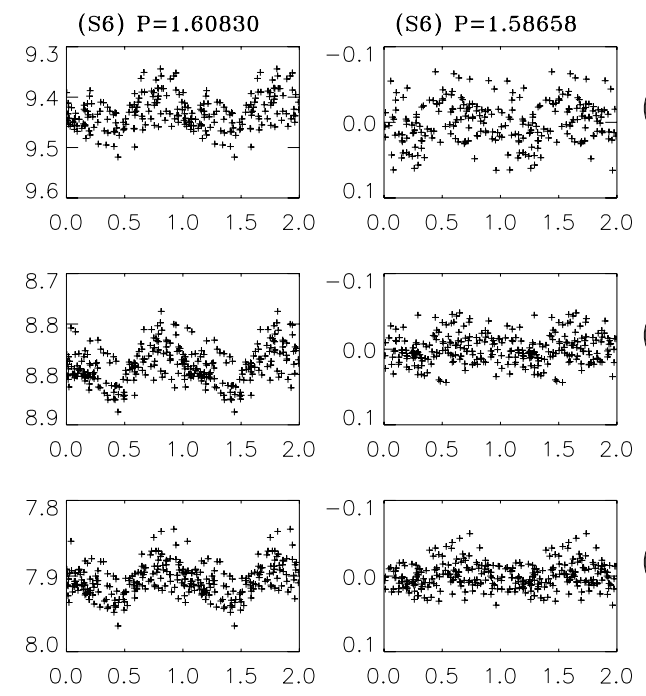

Fig. 4. PPDs of season S6. See text and Fig. 2 for explanation.

their PPDs in the U, B and V in Fig. 4. The left three PPDs are created by folding the original data in S6 with the primary period of 1.60830 and the right three are created by folding the first residuals with the second period of 1.58658 . Although the right three PPDs are not as clear as the left three of the primary period and are worse than the right three PPDs in Fig. 2, they still show a recognizable pattern.

Figure 5 shows the multichannel (UBV) LM spectra for season S7. The top spectrum reveals the primary period of 1.60094. The second spectrum is obtained by subtracting the first period from the top spectrum. Here, the second significant period of 1.61448 appears as the deepest minimum. The third spectrum is obtained by subtracting the second period from the second spectrum. Here, the third period of 1.59542 shows up. The bottom spectrum is obtained by subtracting the third period from the 

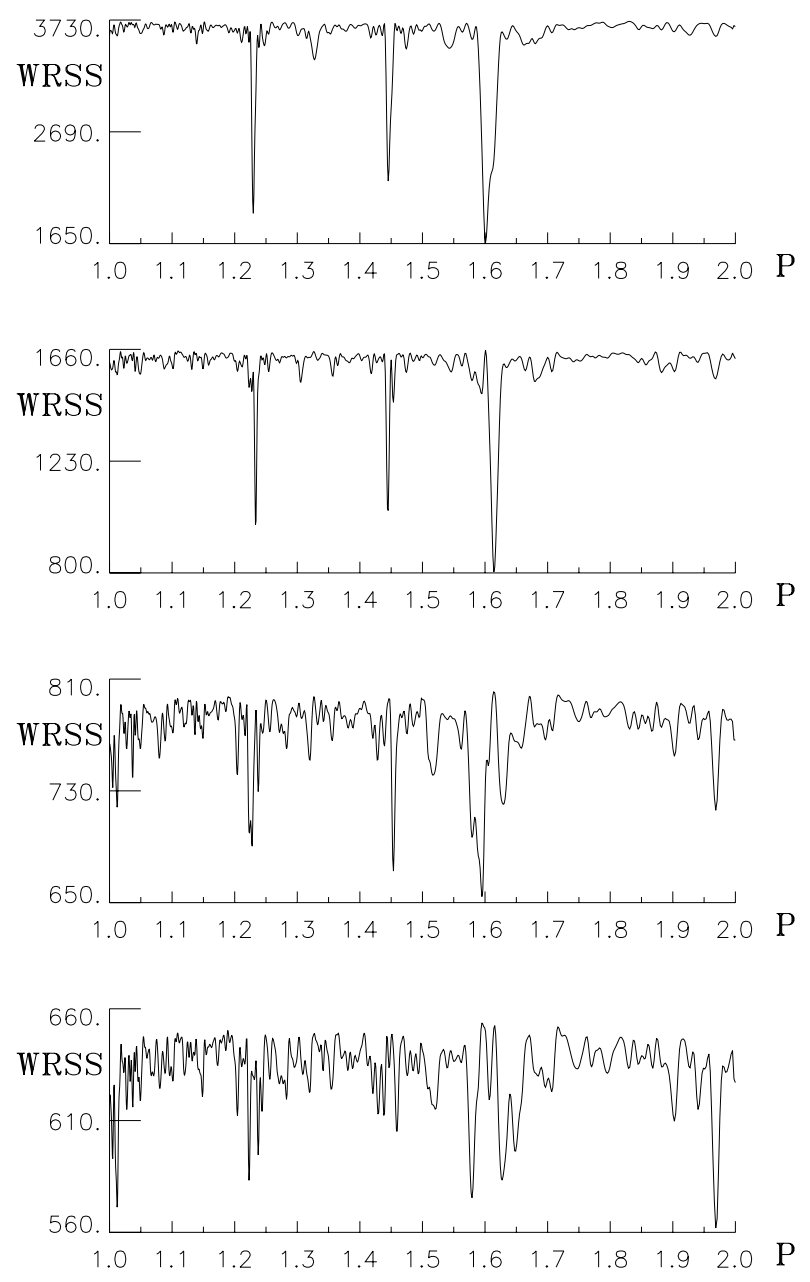

Fig. 5. The multichannel (UBV) LM spectra of season S7. See text and Fig. 1 for explanation.

third spectrum. Here, the minimum around the period of 1.58 has to be checked carefully. After analyzing the original data in S7 and checking the PPDs folded with this period, we identified it as arising from the uneven data sampling and the noise fluctuations. Thus, no significant fourth period is present. S7 is the only season where more than two periods are detected. Besides being checked for the spuriousness arising from the data window, all detected periods are finally supported by their acceptable PPDs.

The PPDs of the U, B and V of S7 of all three periods are given in Fig. 6. All PPDs support the acceptance of all three periods with their clear patterns containing distinguishable maxima and minima. Here, one should notice that dispersions of the residuals in the PPDs do not contain the most important effect the variations arising from the previous more significant periods.

Table 1 gives the results of MPA of LQ Hydrae for each season. All the primary periods $P$ and their error estimates $\sigma$ are optimized with NLM applied to the original data, while the second periods (S2, S6 and S7) are optimized in the first residuals and the third period (S7) in the second residuals. The amplitudes for each period and each channel are obtained from the fits of the multiple period model (if more than one is present), in which the periods are fixed to the optimized values. Since for each period the model contains two harmonics, the amplitudes for channel $c$ are defined as the magnitude differences between the maximum and the minimum of the model curves of that period (if more than one is present).

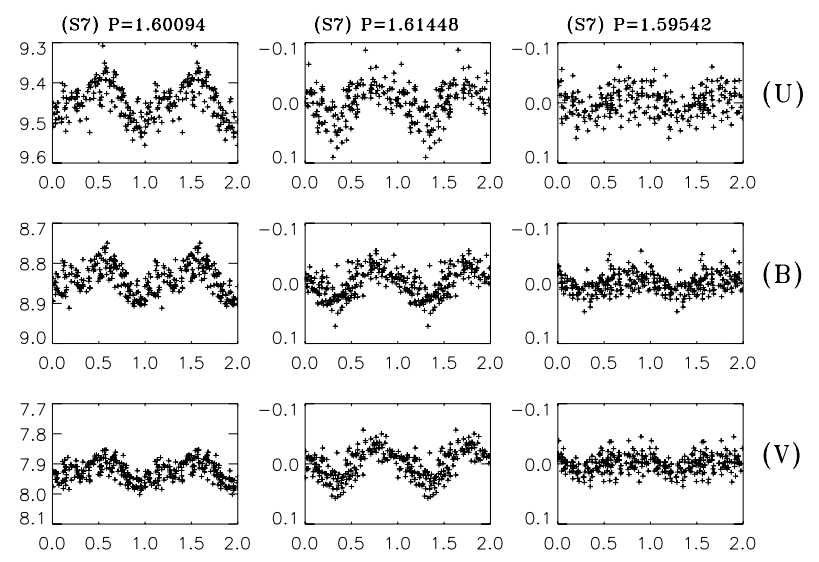

Fig. 6. PPDs of season S7. The left three show the original data folded with the primary period of 1.60094 , the central three are the first residuals folded with the second period of 1.61448, and the right three are the second residuals folded with the third period of 1.59542. See text and Fig. 2 for explanation.

In order to statistically test the significance of the periods shown in Table 1, we applied a bootstrap method for the multichannel UBV data of LQ Hydrae. Our method follows the mathematical formulation by Jetsu \& Pelt (1999). Random permutations were done separately in each individual channel to get a multichannel bootstrapping data sample (the missing observation points in the individual channels were unchanged). To agree with our MPA recovering procedure of the multiple periods, the bootstrap method was applied for each period in the corresponding seasonal data set from which the periods were found, such as the first significant period in the original data, the second period from the first residuals (S2, S6 and S7) and the third period from the second residuals (S7). The bootstrap models for each period are thus identical with the model used in their final NLM optimizations. The statistical significance of our bootstrap sampling results was tested by assuming that the distribution of the resulting period samples follows a scaled Gaussian distribution. In this aspect, the histogram symmetry of the final sampling periods is the main concern. We applied the Kolmogorov-Smirnov test to compute the maximum difference (or the maximum distance $D$ ) between the sample distribution and its corresponding closest scaled Gaussian distribution. The Kolmogorov-Smirnov rejecting criteria $D \geq 0.115$ of 0.01 significant level with 200 samples is used. For each period recovered with MPA, the mean, standard deviation and parameter $D$ from the 200 bootstrapping checks were compiled in the last column in Table 1. The bootstrap check provides further support for our results obtained using the MPA method.

Figure 7 shows all periods with $3 \sigma$ error bars for all seven seasons with the corresponding observing time spans. S2 and S6 have two periods while S7 has three. The primary period appears to vary from season to season within $1.60-1.61$. The second and the third periods are outside this range. All periods are within $1.585-1.625$. Both the shortest period of 1.587 (S6) and the longest period of 1.625 (S2) occur as the second significant period in the seasons. Only in S7, three periods are detected where the third significant period is close to the primary one.

\section{Conclusions and discussion}

1. We have analyzed rotation periods of LQ Hydrae based on the APT data. For the first time three different channels (UBV) have been analyzed simultaneously, with 
Table 1. Observations together with periods and amplitudes of LQ Hydrae. The entries are: beginning and end time of the season (S) as Heliocentric Julian dates $\left(\mathrm{HJD}_{\text {start }}-\mathrm{HJD}_{\text {end }} ; 2440000 .+\right)$ and as calendar months $\left(\mathrm{CD}_{\text {start }}-\mathrm{CD}_{\text {end }}\right)$, time span $T_{\text {span }}$ in days, the number of observations $\left(N^{\mathrm{U}}\right.$, $N^{\mathrm{B}}, N^{\mathrm{V}}$ ) in each channel, the final, refined periods $P$ with their error estimates $\sigma$ from the multichannel (UBV) nonlinear modeling (NLM), and the amplitudes of the single channels U, B, V from the fit of a multiple period model. For comparison, the last column gives the mean, standard deviation and the $D$ parameter (the maximum distance between the sample distribution and its corresponding closest scaled Gaussian distribution) of the bootstrapping result for each period.

\begin{tabular}{cccccccccccc}
\hline \hline $\mathrm{S}$ & $\mathrm{HJD}$ & $\mathrm{CD}$ & $T_{\text {span }}$ & $N^{\mathrm{U}}$ & $N^{\mathrm{B}}$ & $N^{\mathrm{V}}$ & $P \pm \sigma$ & Amp $^{\mathrm{U}}$ & Amp $^{\mathrm{B}}$ & Amp $^{\mathrm{V}}$ & Bootstrap Check \\
\hline 1 & $8919-9122$ & $10 / 1992-5 / 1993$ & 203 & 124 & 170 & 174 & $1.60918 \pm 0.00025$ & 0.072 & 0.080 & 0.079 & $1.60917 \pm 0.00011,0.086$ \\
2 & $9282-9492$ & $10 / 1993-5 / 1994$ & 210 & 89 & 103 & 113 & $1.60263 \pm 0.00024$ & 0.167 & 0.120 & 0.098 & $1.60263 \pm 0.00012,0.097$ \\
& & & & & & & $1.62465 \pm 0.00111$ & 0.032 & 0.033 & 0.030 & $1.62463 \pm 0.00046,0.099$ \\
3 & $10089-10221$ & $1 / 1996-5 / 1996$ & 132 & 36 & 48 & 46 & $1.60914 \pm 0.00104$ & 0.050 & 0.035 & 0.036 & $1.60924 \pm 0.00103,0.083$ \\
4 & $10397-10603$ & $11 / 1996-6 / 1997$ & 216 & 146 & 172 & 170 & $1.60058 \pm 0.00025$ & 0.069 & 0.053 & 0.045 & $1.60058 \pm 0.00014,0.088$ \\
5 & $10731-10968$ & $10 / 1997-6 / 1998$ & 237 & 150 & 177 & 181 & $1.60426 \pm 0.00053$ & 0.036 & 0.032 & 0.031 & $1.60428 \pm 0.00020,0.094$ \\
6 & $11102-11333$ & $10 / 1998-6 / 1999$ & 231 & 125 & 150 & 154 & $1.60830 \pm 0.00043$ & 0.061 & 0.052 & 0.044 & $1.60830 \pm 0.00020,0.091$ \\
& & & & & & & $1.58658 \pm 0.00069$ & 0.037 & 0.017 & 0.017 & $1.58656 \pm 0.00054,0.105$ \\
7 & $11459-11635$ & $10 / 1999-3 / 2000$ & 176 & 135 & 157 & 161 & $1.60094 \pm 0.00034$ & 0.112 & 0.090 & 0.069 & $1.60093 \pm 0.00016,0.091$ \\
& & & & & & & $1.61448 \pm 0.00031$ & 0.058 & 0.050 & 0.055 & $1.61448 \pm 0.00017,0.088$ \\
& & & & & & & $1.59542 \pm 0.00063$ & 0.039 & 0.030 & 0.020 & $1.59545 \pm 0.00034,0.108$ \\
\hline
\end{tabular}

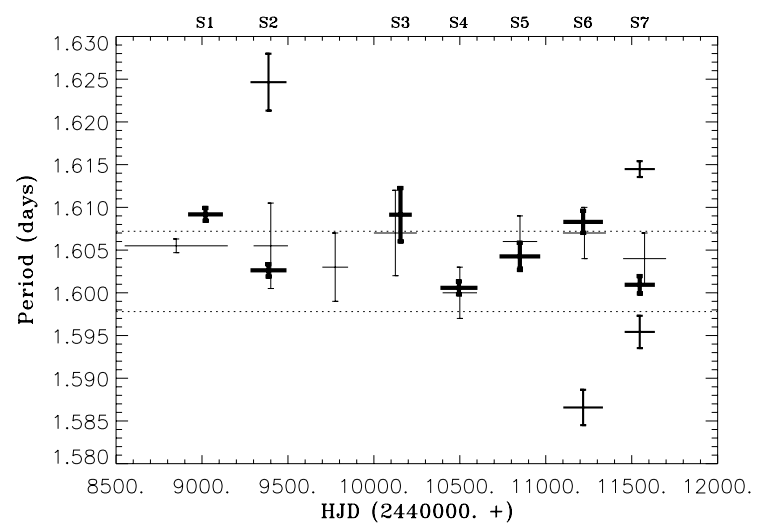

Fig. 7. Periods of LQ Hydrae recovered with MPA from the multichannel (UBV) data. For every season all periods are plotted with a horizontal line whose length corresponds to the time span of the period and a vertical line indicating the $3 \sigma$ error bar. The seven primary periods are plotted with the thickest lines, while the second thickest lines refer to the four additional secondary periods. For comparison, thin lines indicate the periods for the same total time span redrawn from Fig. 7 in Kövári et al. (2004); the two dotted horizontal lines correspond to the pole (upper) and the equator periods (lower) in their model. Note that Kövári et al. periods are from single channel (V) data which explains the larger error bars.

the main conclusion that multiple periods are present. We found two periods in October 1993-May 1994, and October 1998-June 1999, and three periods in October 1999-March 2000. The primary period is present in all seven seasons and varies within the range 1.60-1.61. However, if all the additional secondary periods found in the three seasons are considered, they vary within the range $1.585-1.625$, which increases the range of periods by a factor of four.

The spots responsible for the primary period appear in all observing seasons and thus seem to be permanent. The variation of the primary period indicates that the spots are migrating. The migration could be in latitude, so that differential rotation causes the variability, as it does in the Sun; or it could occur only in longitude at changing rates, without necessarily involving latitude changes. These migration rates can change over time, explaining why we can observe multiple periods at the same time and also why the period

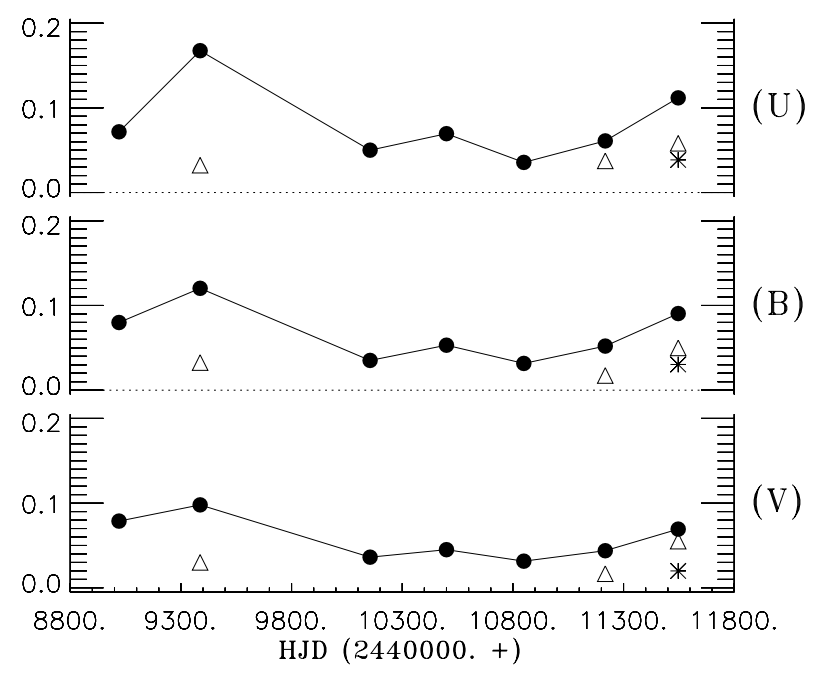

Fig. 8. Amplitudes of the model curves of U, B and V of LQ Hydrae. The full lines connect the dots which are the amplitudes of the primary period. The triangles in the plots are the amplitudes of the second significant periods detected in S2, S6 and S7. The asterisks in the plots are the amplitudes of the third significant period detected in S7. See Tables 1 and Fig. 7.

in different seasons is different. If the change of the primary period arises from the migration in latitude, the responsible spots are located between two latitudes corresponding to two differential rotation rates of 1.60 and 1.61 .

The surface structures responsible for the additional secondary periods seem to be temporary. These structures (a large spot or a spot group) originate either at a certain latitude different from those responsible for the primary period and/or with a different migration rate in longitudes. (See their light curve patterns in Figs. 2, 4 and 6).

2. For the primary period all three channels (U, B and V) show the same amplitude variations (see Fig. 8). The amplitudes (dots in Fig. 8) reach their maxima in October 1993-May 1994 and their minima in October 1997-June 1998. This proves that the spots responsible for the primary period stay permanently as the main tracer for the differential rotation of LQ Hydrae, and also grow and shrink in activity during the seasons. 


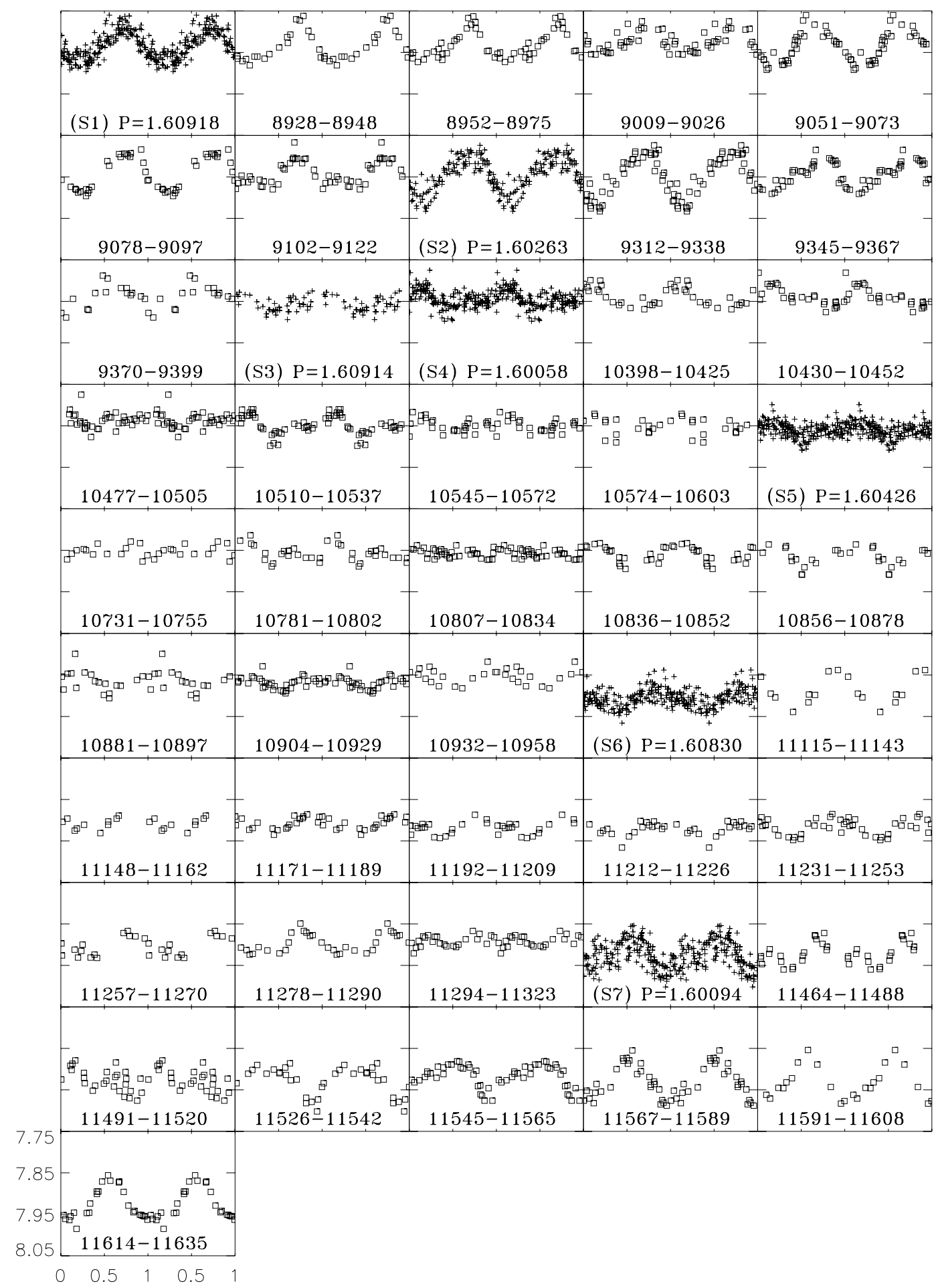

Fig. 9. PPDs of LQ Hydrae in $V$ filter based on the first significant periods recovered with MPA in various seasons (Table 1). The PPDs of the seven seasons are given with their (S) number and period, and plotted with crosses. The PPDs plotted with squares correspond to the sub-divisions of the season folded with the same period. The time spans (HJD $2440000 .+$ ) are given for each of these sub-divisions. All phases in the PPDs have been computed with $\phi=0$ at HJD $=2440000$. The axis ranges for all PPDs are identical to those in the last panel at the bottom.

The amplitudes of the additional periods (triangles and asterisks) are always smaller than those of the primary period. Their periods also always fall outside the range of the variations of the primary period. If the amplitude variations are due to the change of projected area of the surface structure when it moves from one position on the stellar surface to another, the latitudes of the additional periods have less projected area. On the other hand, if the change of the amplitudes is caused by the change of the properties of the active zones, the regions causing the additional periods have weaker spot activities. This might follow for example if the spots have smaller sizes or smaller temperature contrasts.

3. The primary period is clearly not a constant with time: the seven seasonal values deduced from the data are not identical within their $3 \sigma$ errors (Fig. 7). The figure shows that the variation appears to be roughly sinusoidal, being 
about a three-year cycle, but the duration of the used data is too short to make a firm conclusion. Messina \& Guinan (2003) also suggest cyclic changes of the period in a range of $P_{\text {rot }}=1.5938-1.6154$, although with a cycle length of 6.2 years. A long time, constant period has been derived as $P_{\text {rot }}=1.601136 \pm 0.000013$ by Jetsu (1993), $P_{\text {rot }}=1.60088 \pm 0.00003$ by Strassmeier et al. (1997), $P_{\text {rot }}=1.601052 \pm 0.000014$ by Berdyugina et al. (2002) and $P_{\text {rot }}=1.60066 \pm 0.00013$ by Kövári et al. (2004). In this paper, we find that the rotational period varies with time so that any constant long-term period would be affected by the star's seasonal physical activities. Thus, we refrain from giving any mean value for the primary period in a longer time interval.

Considering the degree of the star differential rotation, Berdyugina et al. (2002) gave $\Delta \Omega / \Omega=0.002$ with $P_{\min }=$ 1.600905 and $P_{\max }=1.601198$ and, Kövári et al. (2004) gave $\Delta \Omega / \Omega=0.0057$ with the equatorial period $P_{\text {eq }}=$ 1.597. Comparing with the Sun having $\Delta \Omega / \Omega=0.2$, LQ Hydrae shows almost rigid rotation. In our time series analysis, if all the additional secondary periods found are taken into account, the periods vary within the range $1.585-1.625$. The corresponding differential rotation rate becomes $\Delta \Omega / \Omega=0.025$. Kitchatinov \& Rüdiger (1999) gave a slightly smaller value varying from 0.015 to 0.025 (their Fig. 1), based on the dependence of latitudinal differential rotation on rotation period for two spectral types, G2 and K5. Because the value in our paper is estimated from the photometry of the observable starspots, we may conclude that the real differential rotation rate of LQ Hydrae might be higher than 0.025 .

Kövári et al. (2004) also analyzed the V channel seasonal data covering the same time span as our (UBV) data. They plot their computed seasonal periods in their Fig. 7. We measure from the figure their periods corresponding in time with our seasons and plot the periods in our Fig. 7 for a comparison. This shows that our seven primary periods agree very well with their results. However, their error bars are quite large and it is difficult to see the variations in the primary period. With our new analysis, combining all three channels, the errors are sufficiently small to reveal unambiguous period variations. The comparison shows that our MPA is not only able to constrain the primary period with a higher accuracy, but also allows us to detect additional weaker periods when combining all multichannel data.

4. Figure 9 shows the PPDs for all seven seasons and their sub-divisions in V. Because the constancy of the period in a long run does not agree with our MPA results, we use the primary period in the respective seasons for folding the data. For each of the seven seasons all the data are plotted, while for their sub-divisions only those data are shown which have a sufficient number of observations over about $10-20$ cycles of 1.6 . One should notice that the PPDs of S2, S6, S7 and their sub-divisions include the variations arising from the additional secondary periods. The variations in the PPDs of the sub-divisions of $\mathrm{S} 1, \mathrm{~S} 3, \mathrm{~S} 4$ and $\mathrm{S} 5$ indicate the instability of the responsible spots. The lowest amplitudes occur among the sub-divisions of S5, especially in HJD 24 510 807-24510 834. This factor is also the cause for the large error estimate of the primary period in S5 (see Table 1).

We also performed MPA for all sub-divisions in Fig. 9. Due to the insufficient number of data points, only the primary period can be recovered from these sub-divisions, while their error estimates are so large that they appear to agree with the mean value within their errors. Furthermore, we analyzed all the data together in the full time span of the 7.5 years. Due to the multiple periods, the variations of the periods, their light curve phase shifts, and the long term variation of the mean brightness of the star, no meaningful single period could be derived.

5. This is the second application of MPA (You et al. 2000) to the real observations. Because of the closeness of multiple periods of LQ Hydrae, the original data cannot always be used for optimizing the periods, except for the first significant one. This gives the main difference from the first application of MPA in You \& Duemmler (2005). In the present paper, the LM demonstrates its simplicity and ability to identify the multiple periods by subtracting the most significant minimum in the multichannel spectrum; the NLM shows its ability to constrain the periods with a satisfactory accuracy by one dimensional refinement using the residuals. The statistical test of the significance of the MPA recovered periods is achieved by our multichannel bootstrap analysis. The result shows that the histograms of the bootstrap period samples are usually higher and sharper than the normal Gaussian distribution. In this aspect, we use the corresponding closest scaled Gaussian distribution for checking the histogram symmetry of the bootstrap periods. The Kolmogorov-Smirnov test shows the multichannel bootstrap sampling results match with a scaled Gaussian distribution. This shows that the data sampling of the original observations is not inhomogeneous and the MPA recovered periods are stable against the random noise fluctuations. For the bootstrap periods, their means agree well with these recovered in the MPA and their standard deviations are smaller than the MPA formal error estimates. This is further support for our MPA analysis of LQ Hydrae.

Acknowledgements. The author acknowledges the APT service for the photometry and thanks Dr. Rudolf Duemmler for useful discussions of the topic when commencing this paper in 2001. The author also thanks Eija Laurikainen, Heikki Salo and Juri Poutanen for helping this final submission and the anonymous referee for his valuable comments.

\section{References}

Barnes, J. R., Collier Cameron, A., Donati, J.-F., et al. 2005, MNRAS, 357, L1 Berdyugina, S. V., Pelt, J., \& Tuominen, I. 2002, A\&A, 394, 505

Boyd, L. J., Genet, R. M., \& Hall, D. S. 1984, IAPPP Comm., 15, 20

Boyd, L. J., Genet, R. M., Hall, D. S., Rusby, R. M., \& Henry, G. W. 1990, IAPPP Comm., 42, 44

Croll, B., Walker, G. A. H., Kuschnig, R., et al. 2006, ApJ, 648, 607

Eggen, O. J. 1984, AJ, 89, 1358

Fekel, F. C., Bopp, B. W., Africano, J. L., et al. 1986, AJ, 95, 1150

Jeffers, S. V., Donati, J.-F., \& Collier Cameron, A. 2007, MNRAS, 375, 567

Jetsu, L. 1993, A\&A, 276, 345

Jetsu, L., \& Pelt, J. 1999, A\&AS, 139, 629

Kitchatinov, L. I., \& Rüdiger, G. 1999, A\&A, 344, 911

Kövári, Z., \& Weber, M. 2004, Publ. Astronomy Dept. Eotvos Lorand Univ., 14, 221

Kövári, Z., Strassmeier, K. G., Granzer, T., et al. 2004, A\&A, 417, 1047

McIvor, T., Jardine, M., Cameron, A. C., Wood, K., \& Donati, J.-F. 2004, MNRAS, 355, 1066

Marsden, S. C., Waite, I. A., Carter, B. D., \& Donati, J.-F. 2005, MNRAS, 359, 711

Messina, S., \& Guinan, E. F. 2003, A\&A, 409, 1017

Petit, P., Donati, J.-F., \& Collier Cameron, A. 2004, AN, 325, 221

Strassmeier, K. G., Bartus, J., Cutispoto, G., \& Rodonó, M. 1997, A\&AS, 125, 11

Walker, G. A. H., Croll, B., Kuschnig, R., et al. 2007, ApJ, 659, 1611

You, J., \& Duemmler, R. 2005, A\&A, 433, 297

You, J., Pelt, J., \& Tuominen, I. 2000, A\&AS, 146, 397 